\title{
Polarization Orthogonality for the Co-Existence of Wideband Fading Cognitive Networks
}

\author{
J.-M. Dricot ${ }^{1}$, G. Ferrari ${ }^{2}$, F. Quitin ${ }^{1,3}$, A. Panahandeh ${ }^{1,3}$, Fr. Horlin ${ }^{1}$, and Ph. De Doncker ${ }^{1}$ \\ 1. Université Libre de Bruxelles \\ OPERA Department \\ Wireless Communications Group \\ E-mail: jdricot@ulb.ac.be
}

\begin{abstract}
Orthogonality techniques for cognitive radio networks are important since they enable the primary and secondary terminals to efficiently share the spectral resources in the same location simultaneously. In this paper, we investigate a simple, yet powerful, orthogonality scheme by exploiting the polarimetric dimension. More precisely, we evaluate a scenario where the cognitive terminals use cross-polarized communications in a communication channel subject to wideband (or narrowband) Rayleigh fading. A primary exclusive region in which cognitive terminals are not allowed to transmit is defined and its radius is computed. Finally, the overall performance of the proposed solution is evaluated in terms of network throughput.
\end{abstract}

\section{INTRODUCTION}

Cognitive radio networks and, more generally, dynamic spectrum access networks are becoming a reality. These systems consist of primary nodes, which have guaranteed priority access to spectrum resources, and secondary (or cognitive) nodes, which can re-use the medium in an opportunistic manner [1], [2]. Cognitive nodes are allowed to dynamically operate the secondary spectrum, provided that they do not degrade the primary users' transmissions.

In this paper, we investigate a simple, yet powerful, orthogonality scheme by exploiting the polarimetric dimension [3][5]. More specifically, a dual-polarized wireless channel enables the use of two distinct polarization modes, referred to as co-polar (symbol: $\|$ ) and cross-polar (symbol: $\perp$ ), respectively. Ideally, cross-polar transmissions (i.e., from a transmitting antenna on one channel to the receiving antenna on the corresponding orthogonal channel) should be impossible. In reality, this is not the case due to an imperfect antenna cross-polar isolation (XPI) and a depolarization mechanism that occurs as electromagnetic waves propagate (i.e., a signal sent on a given polarization "leaks" into the other). Both effects combine to yield a global phenomenon referred to as cross-polar discrimination (XPD) [6]-[8].

The scenario of interest for this work is shown in Fig. 1. The primary system consists of a single transmitter located at a distance of $d_{0}$ from its intended receiver. Without any loss of generality, the primary receiver is considered to be located at the origin of the coordinates system, leading to a receivercentric analysis. The secondary (cognitive) terminals are deployed along with the primary ones. However, limitations on

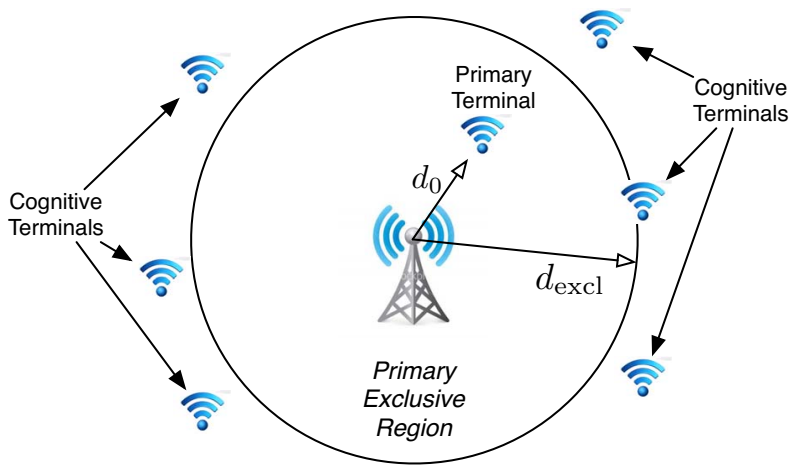

Fig. 1: Cognitive network model: a single primary transmitter is placed at the center of a primary exclusive region (PER), with radius $d_{\text {excl }}$, where its intended receiver is present.

interference prevent them from entering a protected region around the receiver. This region, referred to as the "primary exclusive region" [9], is assumed to be circular and therefore, is completely characterized by its radius, denoted as $d_{\text {excl }}$.

The medium access control (MAC) protocol considered is a variant of the slotted ALOHA protocol [10] such that in each time slot, the nodes transmit independently with a certain fixed probability [11]. This approach is supported by the observations in [12, p. 278] and [11], where it is shown that the traffic generated by nodes using a slotted random access MAC protocol can be modeled by means of a Bernoulli distribution. In fact, in more sophisticated MAC schemes, the probability of transmission of a terminal's transmission can be modeled as a function of more general parameters but since the impact of these parameters is not the focus of the this study, for more details we refer the interested reader to the existing studies in the literature [13], [14].

The remainder of this paper is organized as follows. In Section II, we provide the reader with a theoretical framework that analyzes under what conditions the primary and the secondary users can co-exist in a wideband, dual-polarized network. Then, in Section III, we evaluate the corresponding performance by computing the dimension of the primary exclusive region and the maximum achievable transmission rate of the secondary network. Section IV concludes the paper. 


\section{Probabilistic Co-EXistence And Interference of DUAL POLARIZED NETWORKS}

Consider the cognitive network shown in Fig. 1 with two types of users: primary and secondary (cognitive). The primary network is supposed to be co-polar and the cognitive network is cross-polar. Without cognitive users, the primary network would operate with background noise and with the usual interference generated by the other primary users. Let $\mathbf{C}_{p}$ (dimension: [bit/s/Hz]) be the desired capacity for a user in the primary network ${ }^{1}$. We impose that the secondary network operates under the following outage constraint on a primary user:

$$
\mathbb{P}\left\{\mathbf{C}_{\mathrm{p}} \leq C\right\} \leq \varepsilon
$$

where $0<\varepsilon<1$ and $C$ (dimension: [bit/s/Hz]) is a minimum per-primary user capacity. Equivalently, this constraint guarantees a primary user a maximum transmission rate of at least $C$ for at least a fraction $(1-\varepsilon)$ of the time. Under the simplifying assumption of Gaussian signaling, the rate of this primary user can be written as a function of the signal-to-noise and interference ratio (SINR) as follows:

$$
\mathbf{C}_{\mathrm{p}}=\log _{2}(1+\mathbf{S I N R}) .
$$

and the condition (1) can be equivalently rewritten as

$$
\mathbb{P}\left\{\mathbf{C}_{\mathrm{p}} \leq C\right\} \leq \varepsilon \quad \Leftrightarrow \quad \mathbb{P}\{\text { SINR }>\theta\}>1-\varepsilon
$$

where $\theta \triangleq 2^{C}-1$ and $\mathbb{P}\{$ SINR $>\theta\}$ can be interpreted as the primary link probability of successful transmission for an outage SINR value $\theta$.

In the presence of a generic transmission channel experiencing wideband fading, the transmitted symbols of the considered packet experiences interference of the other symbols that have been delayed by multipath [15]. This phenomenon is referred to as Inter-Symbol-Interference (ISI) and it depends on the channel model, modulation format, and symbol sequence characteristics, among others [16]-[18]. Therefore, the expression of the ISI is hard to obtain and typically is not in closed form. In the network-level approach we follow in this paper, a reasonable approximation can be obtained by considering the ISI as an additive, uncorrelated, Rayleighfaded noise power proportional to the received power [17]. Therefore, the link-level SINR is:

$$
\mathbf{S I N R} \triangleq \frac{\mathbf{P}_{0}\left(d_{0}\right)}{N_{0} B+\mathbf{P}_{\text {int }}+\mathbf{P}_{\text {ISI }}}
$$

where $\mathbf{P}_{0}\left(d_{0}\right)$ is the instantaneous received power (dimension: [W]) at distance $d_{0}, N_{0} / 2$ is the power spectral density of the noise (dimension: [W/Hz]), $B$ the channel bandwidth, and $\mathbf{P}_{\text {int }}$ is the cumulated interference power (dimension: [W]) at the receiver, that is, the sum of the received powers from all the undesired transmitters. Finally, $\mathbf{P}_{\text {ISI }}$ is noise power associated with the ISI. Its average value (noted $P_{\mathrm{ISI}}=\mathbb{E}\left[\mathbf{P}_{\mathrm{ISI}}\right]$ ) is proportional to the received power [17] and it is defined as

$$
P_{\mathrm{ISI}} \triangleq \nu P_{0}\left(d_{0}\right) \quad 0 \leq \nu<1
$$

Note that $\nu=0$ refers to a narrowband scenario. We now

\footnotetext{
${ }^{1}$ In this manuscript, bold letters refer to random variables.
}

provide the reader with a series of theoretical results, which stem from the following theorem.

THEOREM 1 In a wideband, Rayleigh, block-faded, dualpolarized network, where nodes transmit with probability $q$ on the cross-polar channel, the probability that the SINR exceeds a given value $\theta$ on a primary transmission, given a fixed transmitter-receiver distance $d_{0}, N_{\mathrm{int}}^{\perp}$ cross-polar interferers at distances $\left\{d_{i}\right\}_{i=1}^{N_{\mathrm{int}}^{\perp}}$ transmitting at powers $\left\{P_{i}^{\perp}\right\}_{i=1}^{N_{\mathrm{int}}^{\perp}}$ with a cross-polar discrimination coefficient $X P D_{0}$, is

$$
\begin{aligned}
\mathbb{P}\{\text { SINR }> & \theta\}=\exp \left(-\theta \frac{N_{0} B}{P_{0} d_{0}^{-\alpha}}\right) \times\left(\frac{1}{1+\nu \theta}\right) \\
\times & \prod_{i=1}^{N_{\text {int }}^{\perp}}\left\{1-\frac{\theta q}{X P D_{0} d_{i}^{-\beta} \frac{P_{0}}{P_{i}^{\perp}}\left(\frac{d_{i}}{d_{0}}\right)^{\alpha}+\theta}\right\}
\end{aligned}
$$

where $P_{0}$ is the transmit power, $N_{0} B$ is the average power of the background noise, $\theta$ is the SINR threshold, $\alpha$ is the path loss exponent, $X P D_{0}$ is the initial cross-polar discrimination of the antenna, $\beta$ characterizes for the polarization loss over distance, and $\nu$ is the fraction of ISI power.

PROOF: The instantaneous received power $\mathbf{P}(d)$ from a node is exponentially distributed [15] with temporal-average received power $\mathbb{E}_{t}[\mathbf{P}(d)]=P(d)=P . L(d)$, where $P$ denotes the transmit power and $L(d) \propto d^{-\alpha}$ is the path loss at distance $d$ (it accounts for the antenna gains and carrier frequency). Since the received power is Rayleigh faded, its probability density function is:

$f_{\mathbf{P}}(x)=\frac{1}{P(d)} \exp \left\{-\frac{x}{P(d)}\right\}=\frac{1}{P \cdot L(d)} \exp \left\{-\frac{x}{P \cdot L(d)}\right\}$.

In a dual-polarized system, the cross-polar discrimination (XPD) is defined as the ratio of the temporal-average power emitted on the cross-polar channel and the temporal-average power received in the co-polar channel [3], i.e.:

$$
P^{(\perp \rightarrow \|)}(d)=\frac{P^{\perp}(d)}{\operatorname{XPD}(d)}
$$

where $d$ is the transmission distance, $P_{i}^{(\perp \rightarrow \|)}(d) \triangleq$ $\mathbb{E}_{t}\left[\mathbf{P}^{(\perp \rightarrow \|)}(d)\right]$ is the temporal-average value of the instantaneous leaked power $\mathbf{P}^{(\perp \rightarrow \|)}(d)$, and $P^{\perp}(d) \triangleq \mathbb{E}_{t}\left[\mathbf{P}^{\perp}(d)\right]$ is the temporal-average value of the instantaneous cross-polar power $\mathbf{P}^{\perp}(d)$. In a generic situation, the XPD is subject to spatial variability [7] and, therefore, in the context of this network-level analysis, we will define the XPD in a spatialaverage sense, i.e.:

$$
\mathrm{XPD}(d) \triangleq\left\langle\frac{P^{\perp}(d)}{P^{(\perp \rightarrow \|)}(d)}\right\rangle
$$

where the operator $\langle X\rangle$ denotes the average of the value $X$ computed on multiple different locations at the same distance $d$. It is shown in [5]-[7], that $\operatorname{XPD}(d)$, defined according to (6), can be expressed as follows:

$$
\mathrm{XPD}(d)=\mathrm{XPD}_{0} G\left(d, d_{\text {ref }}\right)
$$


where $\mathrm{XPD}_{0} \geq 1$ is the $\mathrm{XPD}$ value at a reference distance $d_{\text {ref }}$ and the function $G\left(d, d_{\text {ref }}\right) \propto d^{-\beta}$ characterizes the de-polarization experienced over the distance. For instance, extensive measurements [7] showed that $\beta \approx 0.4$ at $2.5 \mathrm{GHz}$.

Let the traffic at the $N_{\text {int }}^{\perp}$ cognitive interfering nodes be modeled through the use of independent indicators $\left\{\Lambda_{i}\right\}_{i=1}^{N_{\text {int }}^{\perp} \text {, }}$ with $\forall i ; \Lambda_{i} \in\{0,1\}$, where $\Lambda_{i}=1$ denotes that the $j$-th node is transmitting and $\Lambda_{i}=0$ that it is silent. We assume that the traffic distribution is the same at all interfering nodes of the network, i.e., $\forall i, \mathbb{P}\left\{\Lambda_{i}=1\right\}=q$, which is supported by the analyses presented in [11], [19]. The overall interference power due to the secondary terminals is :

$$
\mathbf{P}_{\text {int }}=\sum_{i=1}^{N_{\text {int }}^{\perp}} \mathbf{P}_{i}^{(\perp \rightarrow \|)}\left(d_{i}\right) \Lambda_{i}
$$

where $\left\{\mathbf{P}_{i}^{(\perp \rightarrow \|)}\left(d_{i}\right)\right\}$ are the (instantaneous) interfering powers at the receiver. The probability that the SINR at the receiver exceeds a given value $\theta$ is:

$\mathbb{P}\{\mathbf{S I N R}>\theta\}=\mathbb{E}\left[\mathbb{P}\left\{\mathbf{P}_{0}>\theta\left(N_{0} B+\mathbf{P}_{\text {int }}+\mathbf{P}_{\text {ISI }}\right)\right\} \mid \mathbf{P}_{\text {int }}, \mathbf{P}_{\text {ISI }}\right]$ $=\mathbb{E} \exp \left(-\frac{\theta}{P_{0} L\left(d_{0}\right)}\left(N_{0} B+\sum_{i=1}^{N_{\text {int }}^{\perp}} \mathbf{P}_{i}^{(\perp \rightarrow \|)} L\left(d_{i}\right) \Lambda_{i}+\mathbf{P}_{\text {ISI }}\right)\right)$

Since all terminals have an independent transmission behavior and all channels are independent, i.e., $\left\{\mathbf{P}_{i}^{(\perp \rightarrow \|)}\right\},\left\{\Lambda_{i}\right\}$ are independent sets of random variables and

$$
\begin{aligned}
\mathbb{P}\{\text { SINR }>\theta\} & =\exp \left(-\theta \frac{N_{0} B}{P_{0} L\left(d_{0}\right)}\right) \times \mathbb{E}\left[\exp \left(-\frac{\theta \mathbf{P}_{\text {ISI }}}{P_{0} L\left(d_{0}\right)}\right)\right] \\
& \times \prod_{i=1}^{N_{\text {int }}^{\perp}} \mathbb{E}\left[\exp \left(-\frac{\theta \mathbf{P}_{i}^{(\perp \rightarrow \|)}\left(d_{i}\right) \Lambda_{i}}{P_{0} L\left(d_{0}\right)}\right)\right] .
\end{aligned}
$$

In this relation, the expectation of the term containing the ISI power is given by:

$\mathbb{E}\left[\exp \left(-\frac{\theta \mathbf{P}_{\text {ISI }}}{P_{0} L\left(d_{0}\right)}\right)\right]=\int_{0}^{\infty} \exp \left(-\frac{\theta x}{P_{0} L\left(d_{0}\right)}\right) f_{\mathbf{P}_{\text {ISI }}}(x) \mathrm{d} x$ and, since the ISI is Rayleigh faded, one has

$$
f_{\mathbf{P}_{\text {ISI }}}(x)=\frac{1}{\nu P_{0} L\left(d_{0}\right)} \exp \left\{-\frac{x}{\nu P_{0} L\left(d_{0}\right)}\right\}
$$

which finally gives

$$
\mathbb{E}\left[\exp \left(-\frac{\theta \mathbf{P}_{\text {ISI }}}{P_{0} L\left(d_{0}\right)}\right)\right]=\frac{1}{1+\nu \theta} .
$$

The last expectation term in (7) can be expressed using (5):

$$
\begin{gathered}
\mathbb{E}\left[\exp \left(-\frac{\theta \mathbf{P}_{i}^{(\perp \rightarrow \|)}\left(d_{i}\right) \Lambda_{i}}{P_{0} L\left(d_{0}\right)}\right)\right]=\mathbb{P}\left\{\Lambda_{i}^{\prime}=1\right\} \\
\times \int_{0}^{\infty} \exp \left(-\frac{\theta p_{i}}{P_{0} L\left(d_{0}\right)}\right) f_{\mathbf{P}_{i}^{(\perp \rightarrow \|)}}\left(p_{i}\right) \mathrm{d} p_{i}+\mathbb{P}\left\{\Lambda_{i}=0\right\} \times 1 \\
=1-\frac{\theta q}{\mathrm{XPD}_{0} d^{-\beta} \frac{P_{0}}{P_{i}^{\perp}}\left(\frac{d_{i}}{d_{0}}\right)^{\alpha}+\theta} .
\end{gathered}
$$

By plugging (8) and (9) into (7), one finally obtains expression (4) for the probability of successful transmission.

Theorem 1 gives interesting insights on the expected performance in a dual-polarized transmission subject to intersymbol and inter-node interference. First, the leftmost term of the expression at the right-hand side of (4) is relevant in a situation where the throughput is limited by the background noise. In large and/or dense networks, the transmission is only limited by the interference and this term is neglectable.

The second and the third terms of expression (4) relate to the ISI interference and the interference generated by the surrounding nodes transmitting in cross-polarized channels, respectively. These terms depend on (i) the polarization characteristics of the interfering nodes, (ii) the traffic density, and (iii) the topology of the network. Note that the impact of the topology has been largely investigated in [20] and we will limit our study to the impact of polarization.

\section{Performance Analysis}

In the following section, we consider (without loss of generality) a scenario made of a single primary emitter subject to interference from a single secondary transmitter using crosspolarized transmissions.

\section{A. Primary Exclusive Region}

As previously anticipated in Section I, the primary exclusive region is completely characterized by the primary exclusive distance $d_{\text {excl }}$, i.e., the minimum distance at which a cognitive terminal has to be, with respect to a primary receiver, so that it does not impact the capacity of the primary user, in a probabilistic sense [9].

LEMMA 2 There exists a region of space, referred to as the primary exclusive region, where the cognitive terminals are not allowed to transmit. This region can be reduced by means of polarimetric orthogonality.

PROOF: Starting from (4), in the presence of a single crosspolar interferer, one can write

$$
\frac{1}{1+\nu \theta}\left(1-\frac{\theta q}{\operatorname{XPD}(d) \frac{P_{0}}{P}\left(\frac{d}{d_{0}}\right)^{\alpha}+\theta}\right)>1-\varepsilon .
$$

This relation is equivalent to

$$
\frac{d}{d_{0}} \geq\left(\frac{1}{\mathrm{XPD}_{0} d^{-\beta}}\right)^{1 / \alpha}\left(\theta \frac{P}{P_{0}} \frac{q-\varepsilon_{\mathrm{wb}}}{\varepsilon_{\mathrm{wb}}}\right)^{1 / \alpha} \triangleq \frac{d_{\mathrm{excl}}}{d_{0}}
$$

where we introduced $\varepsilon_{\mathrm{wb}} \triangleq 1-(1+\nu \theta)(1-\varepsilon)$ which shifts the value $\varepsilon$ to account for the impact of ISI on the links. Note that in the narrowband case, $\nu=0$ and $\varepsilon_{\mathrm{wb}}=\varepsilon$. The definition at the right-hand side of (11) allows to express the minimum distance $d_{\text {excl }}$ as a function of the distance $d_{0}$ and the other main system parameters as follows:

$$
d_{\mathrm{excl}}=d_{0}\left(\frac{1}{\mathrm{XPD}_{0} d^{-\beta}}\right)^{1 / \alpha}\left(\theta \frac{P}{P_{0}} \frac{q-\varepsilon_{\mathrm{wb}}}{\varepsilon_{\mathrm{wb}}}\right)^{1 / \alpha} .
$$




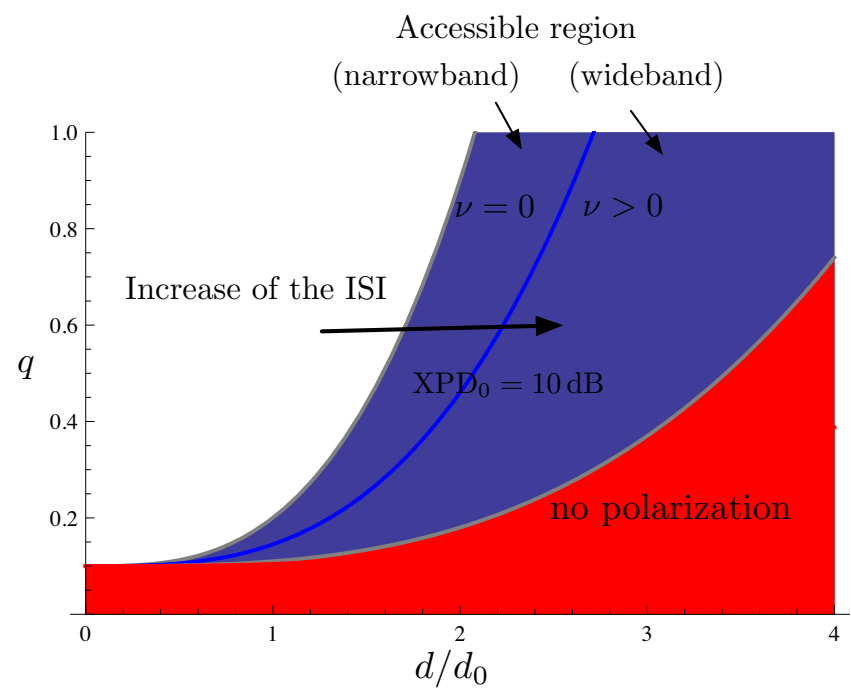

Fig. 2: Accessible throughput of the secondary terminals as a function of the primary-to-secondary distances ratio. The parameter value $\nu=0$ and $\nu>0$ correspond to narrowband scenarios and wideband scenario, respectively.

Furthermore, since $\alpha \geq 2$, using polarization orthogonality, i.e., causing $\mathrm{XPD}_{0}>1$, reduces $d_{\mathrm{excl}}$.

It can be observed that in the case without polarization (i.e., with $\mathrm{XPD}_{0} d^{-\beta}=1$ ), it is very likely that $d_{\text {excl }} \gg d_{0}$, i.e., the cognitive terminals must be located outside the transmission zone defined by the primary emitter-receiver distance. Also, the exclusive distance increases as a function of the terminal probability of transmission but its gradient is smaller in the dual-polarized case since $1 /\left(\mathrm{XPD}_{0} d^{-\beta}\right) \leq 1$.

It is interesting to observe that relation (10) can also be used to parameterize practical realizations of the antennas. Indeed, it yields that

$$
\mathrm{XPD}(d) \geq \frac{P}{P_{0}}\left(\frac{d_{0}}{d}\right)^{\alpha} \frac{\left(q-\varepsilon_{\mathrm{wb}}\right)}{\varepsilon_{\mathrm{wb}}} \theta
$$

from which, being $\mathrm{XPD}(d)=\mathrm{XPD}_{0} d^{-\beta}$, it follows:

$$
\mathrm{XPD}_{0} \geq d^{\beta} \frac{P}{P_{0}}\left(\frac{d_{0}}{d}\right)^{\alpha} \frac{\left(q-\varepsilon_{\mathrm{wb}}\right)}{\varepsilon_{\mathrm{wb}}} \theta
$$

Therefore, the quantity at the right-hand side of (12) represents the minimum amount of XPD that the antenna of the cognitive terminal must possess. This value depends on the network configuration but also on the propagation environment (through the de-polarization modeled by means of the parameter $\beta$ ).

Finally, regarding a comparison between narrowband and wideband systems, it can be noted that

$$
\frac{1}{1+\nu \theta} \leq 1 \quad \forall \nu \in[0 ; 1]
$$

which implies that the presence of ISI impacts negatively the link probability of outage. This in turn results in (i) an increase of the primary exclusive region and (ii) a degradation of system throughput. More precisely, the Fig. 2 presents a comparison of the accessible transmission regions in the narrowband fading and wideband fading cases. The system parameters are $\theta=10 \mathrm{~dB}, \mathrm{XPD}_{0}=10 \mathrm{~dB}, \alpha=2$, and $\beta=0.4$.

LEMMA 3 If $q<\varepsilon_{w b}$, polarization orthogonality is not required to achieve a probabilistic co-existence.

PROOF: As previously introduced, the coefficient $\mathrm{XPD}_{0}$ of a dual-polarized system is greater than or equal to 1 . Also, the minimum value of $\mathrm{XPD}_{0}$ to guarantee error-free transmissions on the cross-polar channel, given in (12). Therefore, the initial value of the XPD always verifies

$$
\mathrm{XPD}_{0}=\max \left\{1, d^{\beta} \frac{P}{P_{0}}\left(\frac{d_{0}}{d}\right)^{\alpha} \frac{\left(q-\varepsilon_{\mathrm{wb}}\right)}{\varepsilon_{\mathrm{wb}}} \theta\right\} .
$$

Finally, since in (12) all quantities are greater than zero, having $q<\varepsilon_{\mathrm{wb}}$ causes the quantity $q-\varepsilon_{\mathrm{wb}}$ to be negative and the solution of (13) is given by $\mathrm{XPD}_{0}=1$.

Lemma 3 indicates that, if the desired throughput of the secondary network remains limited, then the minimum required capacity is guaranteed on the primary system without summoning up the orthogonality of polarization. Therefore, the cross-polar channel can be kept available for other terminals that may require higher data rates.

\section{B. Probabilistic Link Throughput}

Referring back to our definition of the probabilistic coexistence of the primary and secondary terminals in (1), a transmission is said to be successful if and only if the primary terminal is not in an outage for a fraction of time longer than $(1-\varepsilon)$, i.e., if the (instantaneous) SINR of the cognitive terminal is above the threshold $\theta$. We denote this probability of successful transmission in a primary link as

$$
\mathcal{P}_{s}=\mathbb{P}\{\mathbf{S I N R}>\theta\} .
$$

The probabilistic link throughput [21] (adimensional) of a primary terminal is defined as follows:

- in the full-duplex communication case, it corresponds to the product of (i) $\mathcal{P}_{s}$ and (ii) the probability that the transmitter actually transmits (i.e., $q$ );

- in the half-duplex communication case, it corresponds to the product of (i) $\mathcal{P}_{s}$, (ii) the probability that the transmitter actually transmits (i.e., $q$ ), and (iii) the probability that the receiver actually receives (i.e., $1-q$ ).

The probabilistic link throughput can be interpreted as the unconditioned reception probability which can be achieved with a simple automatic-repeat-request (ARQ) scheme with error-free feedback [22]. For the slotted ALOHA transmission scheme under consideration, the probabilistic throughput in the half-duplex mode is then $\tau^{\text {(half })} \triangleq q(1-q) \mathcal{P}_{s}$ and in full-

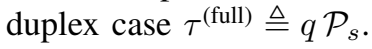

In Fig. 3, the probabilistic link throughput of a primary terminal is presented in scenario as a function of the terminals probability of transmission probability $q .^{2}$ The considered

\footnotetext{
${ }^{2}$ It is recalled that we consider that both the primary and the secondary systems are implementing the same technology and, therefore, the value $q$ corresponds to the terminal probability of transmission of the primary and the secondary terminals.
} 


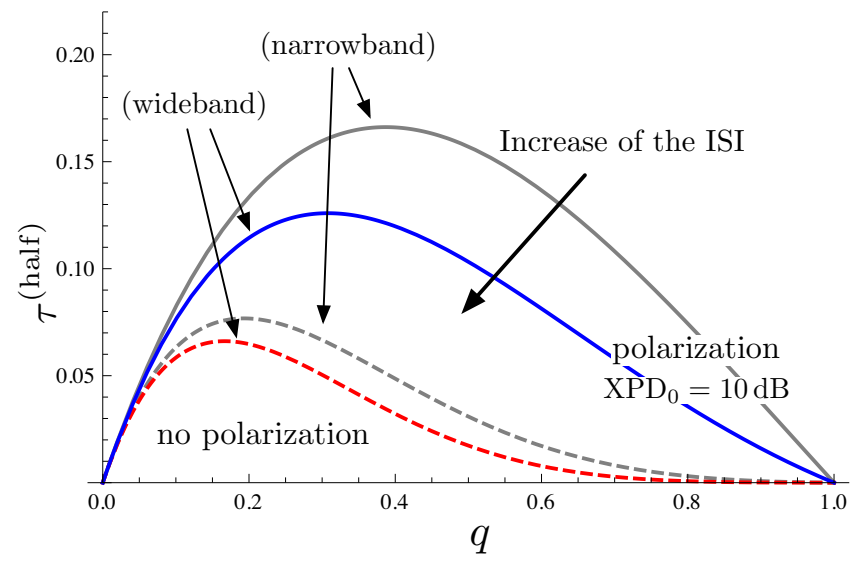

Fig. 3: Impact of the polarization orthogonality on the system-level performance. The parameter value $\nu=0$ and $\nu>0$ correspond to narrowband scenarios and wideband scenario, respectively.

transmission system is half-duplex. The other parameters are $P=P_{0}, N_{\text {int }}^{\perp}=5, \beta=0.4, \alpha=2, d_{0}=150 \mathrm{~m}$, and $d_{\{1,2,3,4,5\}}=250 \mathrm{~m}$. It can be observed that the polarization orthogonality has a string beneficial impact on the link throughput. More precisely, when the terminal probability of transmission becomes significant (e.g., $q \geq 0.1$ ), the link throughput can be the double with respect to a scenario without polarized antennas. As expected, the presence of ISI has a negative impact on the system throughput but, since it equivalently affects the links with and without polarized antennas, the use of polarization still presents an interesting technique to support heterogeneous system co-existence.

\section{CONCLUSIONS}

In this paper, we have presented a novel theoretical framework to demonstrate the network-level performance increase that can be achieved in a polarimetric orthogonality-oriented system subject to Rayleigh fading and probabilistic coexistence of primary and secondary (cognitive) networks.

Our results suggest that dual-polarized networks are of interest, even if orthogonality (indicated by the XPD value) is limited. Indeed, with respect to the classical implementation of probabilistic co-existence of primary and secondary network on the same (single polarization) channel, the use of polarization orthogonality allows to remarkably increase the per-link throughput and reduce the primary exclusive region.

Finally, we are currently investigating distributed detection mechanisms that can be used to infer the polarization state of the terminals in presence.

\section{ACKNOWLEDGMENT}

The support of the Belgian National Fund for Scientific Research (FRS-FNRS) is gratefully acknowledged.

Part of this work was supported by COST Action IC0902 "Cognitive Radio and Networking for Cooperative Coexistence of Heterogeneous Wireless Networks".

\section{REFERENCES}

[1] Q. H. Mahmoud, Ed., Cognitive Networks. New York, USA: Wiley, July 2007.

[2] I. F. Akyildiz, W.-Y. Lee, M. C. Vuran, and S. Mohanty, "NeXt generation/dynamic spectrum access/cognitive radio wireless networks: a survey," Comput. Netw., vol. 50, no. 13, pp. 2127-2159, September 2006.

[3] C. Oestges, "A comprehensive model of dual-polarized channels: From experimental observations to an analytical formulation," in Proc. of the Third International Conference on Communications and Networking in China, Hangzhou, China, August 2008, pp. 1071-1075.

[4] M. Shafi, M. Zhang, A. Moustakas, P. Smith, A. Molisch, F. Tufvesson, and S. Simon, "Polarized MIMO Channels in 3D: Models, Measurements and Mutual Information," IEEE J. Selected Areas Comm., vol. 24, no. 3, pp. 514-527, March 2006.

[5] F. Argenti, T. Bianchi, L. Mucchi, and L. S. Ronga, "Polarization diversity for multiband UWB systems," Signal Process., vol. 86, no. 9, pp. 2208-2220, September 2006.

[6] C. Oestges, V. Erceg, and A. Paulraj, "Propagation modeling of multipolarized MIMO fixed wireless channels," IEEE Trans. Veh. Techn., vol. 53, no. 3, pp. 644-654, May 2004.

[7] V. Erceg, P. Soma, D. S. Baum, and S. Catreux, "Multiple-input multipleoutput fixed wireless radio channel measurements and modeling using dual-polarized antennas at $2.5 \mathrm{GHz}$," IEEE Transactions on Wireless Communication, vol. 1, pp. 396-400, May 2004.

[8] F. Quitin, C. Oestges, F. Horlin, and Ph. De Doncker, "Small-scale variations of cross-polar discrimination in polarized mimo systems," in Proc. of 3rd European Conference on Antennas and Propagation (EuCAP 2009), Germany, March 2009.

[9] M.Vu, N. Devroye, and V. Tarokh, "On the primary exclusive region of cognitive networks," EEE Trans. Wireless Comm., vol. 8, no. 7, pp. $3380-3385$, July 2009.

[10] N. Abramson, "The ALOHA system - another alternative for computer communications," in Proc. Fall Joint Computer Conference, AFIPS Conference, vol. 37, Houston, Texas, USA, November 1970, pp. 281285.

[11] F. Tobagi, "Analysis of a two-hop centralized packet radio networkPart I: slotted ALOHA," IEEE Trans. Commun., vol. 28, no. 2, pp. 196-207, February 1980.

[12] D. Bertsekas and R. Gallager, Data networks (2nd ed.). Upper Saddle River, NJ, USA: Prentice-Hall, Inc., 1992.

[13] Y. Kwon, Y. Fang, and H. Latchman, "Performance analysis for a new medium access control protocol in wireless LANs," Wirel. Netw., vol. 10, no. 5, pp. 519-529, September 2004.

[14] H.-Y. Hsieh and R. Sivakumar, "IEEE 802.11 over multi-hop wireless networks: problems and new perspectives," in Vehicular Technology Conference, 2002. Proc. (VTC'2002), vol. 2, Vancouver, British Columbia, Canada, September 2002, pp. 748-752.

[15] A. Goldsmith, Wireless Communications. New York, NY, USA: Cambridge University Press, 2005.

[16] J. Chuang, "The effects of time delay spread on portable radio communications channels with digital modulation," IEEE J. Selected Areas Commun., vol. SAC-5, no. 5, pp. 879-889, June 1987.

[17] S. Gurunathan and K. Feher, "Multipath simulation models for mobile radio channels," in Proc. IEEE Vehic. Technol. Conf., May 1992, pp. $131-134$.

[18] V. Fung, R. Rappaport, and B. Thoma, "Bit error simulation for $\pi / 4$ DQPSK mobile radio communication using two-ray and measurement based impulse response models," IEEE J. Select. Areas Commun., vol. 11, no. 3, pp. 393-405, April 1993.

[19] R. Nelson and L. Kleinrock, "The spatial capacity of a slotted ALOHA multihop packet radio network with capture," IEEE Trans. Commun., vol. 32, no. 6, pp. 684-694, June 1984.

[20] O. Dousse, F. Baccelli, and P. Thiran, "Impact of interferences on connectivity in ad hoc networks," IEEE/ACM Trans. Netw., vol. 13, no. 2, pp. 425-436, April 2005.

[21] M. Haenggi, "Outage, Local Throughput, and Capacity of Random Wireless Networks," IEEE Transactions on Wireless Communications, vol. 8, no. 8, pp. 4350-4359, August 2009.

[22] S. Ahmed and M. S. Alam, "Performance evaluation of important ad hoc network protocols," EURASIP Journal on Wireless Communications and Networking, vol. 2, pp. 46-57, April 2006. 